\title{
MicroRNA-29c inhibits proliferation and promotes apoptosis in non-small cell lung cancer cells by targeting VEGFA
}

\author{
SHIJUAN ZHAN ${ }^{1}$, CHUNFENG WANG ${ }^{2}$ and FANGQING YIN ${ }^{3}$ \\ ${ }^{1}$ Department of Oncology, Linyi People's Hospital; ${ }^{2}$ Department of Medicine, Health School of Linyi, \\ Linyi, Shandong 276000; ${ }^{3}$ Department of Pediatric Surgery, Linyi People's Hospital, Linyi, Shandong 276003, P.R. China
}

Received June 15, 2017; Accepted February 6, 2018

DOI: $10.3892 / \mathrm{mmr} .2018 .8678$

\begin{abstract}
Non-small cell lung cancer (NSCLC) is a primary sub-type of lung cancer with a high incidence rate and poor prognosis. The primary therapeutic treatment for NSCLC is chemotherapy, which is considered to be ineffective and excessively toxic. Novel therapeutic methods, particularly molecular targeted therapy, have attracted considerable attention. MicroRNAs (miRs) are reported to be potential biomarkers and targeted agents with roles in various types of tumors. Herein, the present study presented the observation of aberrant low expression of miR-29c and associated overexpression of vascular endothelial growth factor $\mathrm{A}$ (VEGFA) in NSCLC tumor tissues. The effects of miR-29c upon NSCLC tumor progression, including cell proliferation and cellular apoptosis, were investigated. The possible regulatory mechanism of action of miR-29c on its direct target VEGFA and the phosphatidylinositol 3-kinase (PI3K)/RAC- $\alpha$ serine/threonine-protein kinase (Akt) signaling pathway was examined using multiple methods, including reverse transcription-quantitative polymerase chain reaction analysis, dual luciferase assay and western blot analysis. The results demonstrated that miR-29c expression was downregulated in NSCLC tumor tissues compared with normal tissues. A marked negative correlation in the expression of miR-29c and VEGFA was observed in clinical NSCLC tissues and cultured NSCLC cells. Overexpression of miR-29c may inhibit cell proliferation and accelerate the cellular apoptosis rate of NSCLC tumor cells. Furthermore, the overexpression of miR-29c was demonstrated to be able to downregulate the expression levels of VEGFA and PI3K/Akt signaling pathway-associated proteins. The results of the present study suggested that miR-29c might regulate NSCLC tumor progression by targeting VEGFA.
\end{abstract}

Correspondence to: Dr Fangqing Yin, Department of Pediatric Surgery, Linyi People's Hospital, 27 Jiefang Road, Lanshan, Linyi, Shandong 276003, P.R. China

E-mail: yinfangqinglyph@163.com

Key words: microRNA-29c, non-small cell lung cancer, vascular endothelial growth factor A

\section{Introduction}

Lung cancer, with a high incidence rate and poor prognosis, is reported to be one of the most frequently-occurring aggressive tumors, and is a principal cause of human mortality around the world (1). Due to the high invasiveness and metastatic ability, the 5-year survival rate of lung cancer is $<15 \%$, which is lower than most common cancers (2). In particular, non-small cell lung cancer (NSCLC) accounts for $>80 \%$ of all lung cancer cases (3). Although it is frequently considered to be ineffective or excessively toxic, the primary therapeutic treatment for NSCLC is chemotherapy (4). Intensive research is required to identify novel, effective and low-toxicity therapeutic methods. Given the development of targeted agents which are able to target specific molecular pathways in tumor cells, molecular targeted therapy has become a promising therapeutic option for various types of cancer. Thus, research into specific targets for NSCLC has become a focus in recent decades.

MicroRNAs (miRNAs/miRs) are single-stranded small ( 24 nucleotides) non-coding endogenous RNAs that regulate the expression of genes in a variety of cellular processes, by binding to the 3' untranslated region (UTR) of their target mRNAs for degradation and translation suppression (5). A previous study indicated the important roles of miRNAs in carcinogenesis (6). The miR-29 family is aberrantly expressed in NSCLC cells. According to a previous study, the miR-29 family serves a notable role in lung tumor cellular processes associated with poor prognosis (7). miR-29c is a miR-29 family member (7). It was reported that miR-29c was observably underexpressed in NSCLC tumor tissues, illustrating that miR-29c may be able to suppress NSCLC tumorigenesis $(8,9)$.

The growth of tumors is accompanied by stimulation of angiogenesis. One of the primary factors in tumor angiogenesis stimulation is vascular endothelial growth factor A (VEGFA) (10). According to previous research, high expression of VEGF is associated with the proliferation and metastasis of several types of cancer (11). Through bioinformatics methods, it was identified that VEGFA may be a gene target for miR-29c. However, studies focusing on whether miR-29c is able to regulate cell proliferation and cellular apoptosis in NSCLC tumors by targeting VEGFA have not yet been reported.

In the present study, aberrant underexpression of miR-29c and overexpression of VEGFA in NSCLC tumor tissues was observed. Clinical sample investigation and fundamental 
research were combined to examine the correlation between the expression of miR-29c and VEGFA, and the regulatory mechanism of action of miR-29c on NSCLC tumor progression.

\section{Materials and methods}

Specimens. A total of 30 cases of NSCLC tumor tissue samples and the corresponding para-carcinoma tissue samples were obtained from patients with NSCLC who were treated in Linyi People's Hospital from January 2014 to January 2016; there were a total of 17 male and 13 female patients, and the mean age was $51 \pm 14$-years-old. All the collected cases were pathologically diagnosed as NSCLC without any preoperative radiotherapy and/or chemotherapy. The reverse transcription-quantitative polymerase chain reaction (RT-qPCR) method was applied for the measurement of the expression of miR-29c and VEGFA in the collected NSCLC tumor tissue samples and the corresponding para-carcinoma tissue samples. The correlation between miR-29c and VEGFA expression was statistically analyzed. The present study was approved by the ethics committee of Linyi People's Hospital (Linyi, China). Written informed consent was obtained from all patients.

Cell culture. Human NSCLC cell lines including A549, NCI-H1299 and H1650 were cultivated in RPMI-1640 medium (Invitrogen; Thermo Fisher Scientific, Inc., Waltham, MA, USA) with $10 \%$ fetal bovine serum (Invitrogen; Thermo Fisher Scientific, Inc.) at $37^{\circ} \mathrm{C}$ in an incubator $\left(5 \% \mathrm{CO}_{2}\right.$, humidified $)$. The RT-qPCR method was applied for the measurement of the expression of miR-29c in the above cell lines. The cell line with the lowest miR-29c expression was chosen for the following experiments.

Cell transfection. Logarithmic growth phase NCI-H1299 cells were trypsinized, washed and seeded in 96-well plates at a density of $8 \times 10^{3}$ cells/well, prior to being transfected with $10 \mathrm{nM}$ miR-29c mimics or miR-negative control (NC) mimics using Lipofectamine ${ }^{\circledR} 2000$ (Invitrogen; Thermo Fisher Scientific,Inc.) according to the manufacturer's protocols. Cells were separated into three groups: i) Control group; ii) miR-29c mimic group (transfected with miR-29c mimics); and iii) NC group (transfected with miR-NC). Oligonucleotide sequences of miR-29c mimic and miR-NC were as listed: miR-29c mimics forward, 5'-UAGCACCAUUUGAAAUCGGUUA-3', reverse 5'-UAACCGAUUUCAAAUGGUGCUA-3'; miR-NC forward, 5'-UCACAACCUCCUAGAAAGAGUAGA-3' and reverse, 5'-UCUACUCUUUCUAGGAGGUUGUGA-3'. The oligonucleotides were chemosynthesized at Extended Nature Biotechnology Co., Ltd. (Shanghai, China). Total RNA was collected following $72 \mathrm{~h}$. The RT-qPCR method was applied to detect the transfection efficiency in the three groups.

Cell proliferation analysis. A total of $72 \mathrm{~h}$ following cell transfection, an MTT assay (Invitrogen; Thermo Fisher Scientific, Inc.) was used to monitor the cell proliferation rate of each group. NCI-H1299 cells were first washed with buffer (PBS, $\mathrm{pH}$ 7.4), trypsinized, washed, counted and reseeded into a 96-well plate at a density of $1 \times 10^{4}$ cells/100 $\mu \mathrm{l} /$ well. A total of $10 \mu \mathrm{l}$ MTT reagent was added and the plate was cultured at $37^{\circ} \mathrm{C}$ in an incubator $\left(5 \% \mathrm{CO}_{2}\right.$, humidified) until a purple precipitate appeared. Thereafter, $100 \mu \mathrm{l}$ detergent solution (dimethyl sulfoxide) was then used to dissolve the formazan crystals and the plate was incubated at $37^{\circ} \mathrm{C}$ for $2 \mathrm{~h}$ away from light. Absorbance was detected at $570 \mathrm{~nm}$ using a microplate reader.

Cellular apoptosis analysis. A total of $72 \mathrm{~h}$ following cell transfection, flow cytometry with the Annexin V-fluorescein isothiocyanate (FITC)/propidium iodide (PI) staining method was employed to monitor the cellular apoptosis in each group. NCI-H1299 cells were washed, trypsinized and resuspended in the staining solution provided with the Annexin V-FITC Apoptosis Detection kit (Invitrogen; Thermo Fisher Scientific, Inc.), according to the manufacturer's protocol. Following incubation for $1 \mathrm{~h}$ at $37^{\circ} \mathrm{C}$, cellular apoptosis was measured with a flow cytometer (BD Biosciences, Franklin Lakes, NJ, USA). Cells with a positive Annexin V-FITC signal and a negative PI signal were regarded as apoptotic cells. The cell number at each phase was analyzed using FloJo software version 7.6.3 (FloJi LLC, Ashland, OR, USA).

RT-qPCR analysis. Total RNA was extracted from cells using a miRNeasy Mini kit (Qiagen GmbH, Hilden, Germany), according to the manufacturer's protocol. The reverse transcription of VEGFA RNA and miR-29c-3p was conducted using a TaqMan MicroRNA RT kit (Invitrogen; Thermo Fisher Scientific, Inc.) at $42^{\circ} \mathrm{C}$ for $1 \mathrm{~h}$, according to the manufacturer's protocol. miScript SYBR ${ }^{\circledR}$ Green PCR kit (Qiagen, Inc., Valencia, CA, USA) was employed for RT-qPCR analysis, the amplification conditions were: Total volume $20 \mu \mathrm{l}$, initial denaturation $95^{\circ} \mathrm{C}$ for $10 \mathrm{~min}$, then 45 cycles $95^{\circ} \mathrm{C}$ for $15 \mathrm{sec}$, $60^{\circ} \mathrm{C}$ for $1 \mathrm{~min}$, which was performed using a 7900HT Fast Real-Time PCR system (Applied Biosystems; Thermo Fisher Scientific, Inc.). The The sequences of the primers used were: miR-29c-3p forward, 5'-ACACTCCAGCTGGGTAGCACC ATTTGA-3', reverse, 5'-TGGTGTCGTGGAGTCG-3'; U6 forward, 5'-CTCGCTTCGGCAGCACA-3' reverse, 5'-AAC GCTTCACGAATTTGCGT-3'; VEGFA forward, 5'-TTTCTG CTGTCTTGGGTGCATTGG-3', reverse, 5'-ACCACTTCG TGATGATTCTGCCCT-3' and GAPDH forward, 5'-ACACCC ACTCCTCCACCTTT-3' and reverse 5'-TTACTCCTTGGA GGCCATGT-3'. U6 small RNA served as the internal control material for miR-29c-3p, and the relative expression level of VEGFA RNA was normalized to GAPDH using the $2^{-\Delta \Delta C q}$ quantification cycle method (12).

Western blot analysis. Total proteins were extracted from cells using T-PER Protein Extraction Reagent (Invitrogen; Thermo Fisher Scientific, Inc.), according to the manufacturer's protocol. Protein concentration was determined using a Bicinchoninic Acid kit (Beyotime Institute of Biotechnology, Shanghai, China). Proteins (15 $\mu \mathrm{g} / \mathrm{lane})$ were resolved by $10 \%$ SDS-PAGE and transferred to a polyvinylidene fluoride (PVDF) membrane. Subsequently, the PVDF membrane was blocked in 5\% non-fat milk in Tris Buffer Saline containing $0.1 \%$ Tween-20, at room temperature for $1 \mathrm{~h}$ and blotted with antibodies against GAPDH (1:1,000; cat. no. 5174; Cell Signaling Technology, Inc. Danvers, MA, USA), VEGFA (1:1,000; cat. no. 8065; Cell Signaling Technology, Inc.), phosphorylated (p)-PI3K (1:1,000; ab151549; Abcam, Cambridge, MA, USA) and p-Akt (1:1,000; cat. no. 4060; Cell 
A

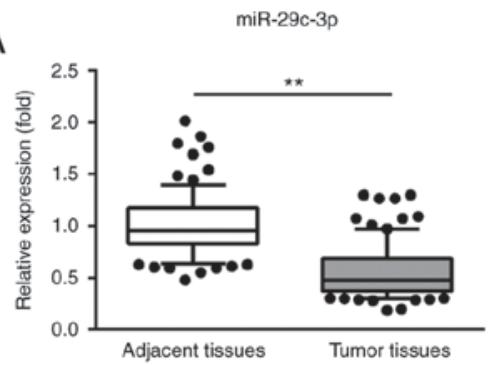

B

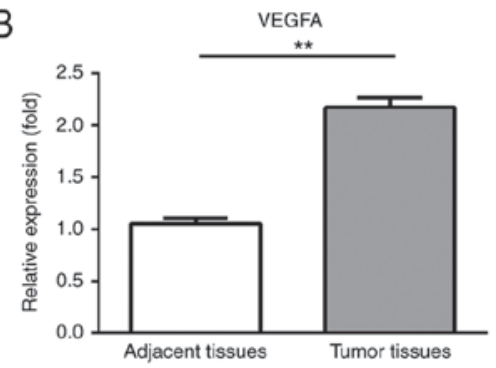

C

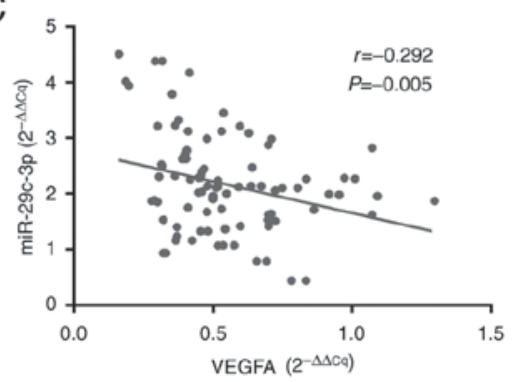

Figure 1. Negative correlation is observed between VEGFA and miR-29c-3p. (A) miR-29c-3p and (B) VEGFA expression in normal and non-small cell lung cancer tumor tissues, and (C) the statistical correlation between miR-29c-3p and VEGFA expression levels. ${ }^{* *} \mathrm{P}<0.01$. miR, microRNA; VEGFA, vascular endothelial growth factor A.

Signaling Technology, Inc.) at $4^{\circ} \mathrm{C}$ overnight. GAPDH served as the internal control material. Chemiluminescence via an Echo-chemiluminescence Detection system (GE Healthcare, Chicago, IL, USA) was measured following incubation with anti-rabbit immunoglobulin G secondary antibody conjugated to horseradish peroxidase $(1: 1,000$; cat. no. 7074; Cell Signaling Technology, Inc.) in room temperature for $2 \mathrm{~h}$. The relative expression of VEGFA, $\mathrm{p}-\mathrm{PI} 3 \mathrm{~K}$ and $\mathrm{p}$-Akt proteins was evaluated statistically by Quantity One 4.6.2 (Bio-Rad Laboratories, Inc., Hercules, CA, USA).

Dual-luciferase reporter analysis. Through bioinformatics methods using TargetScan 7.1 (http://www.targetscan.org), it was identified that VEGFA may be a target gene for miR-29c. Two types of 3'UTR fragments (wild type and mutant type) of VEGFA were inserted into the XhoI-PmeI restriction sites of the 3'UTR of the Renilla luciferase gene of the dual-luciferase reporters psiCHECK2 (Promega Corporation, Madison, WI, USA) to obtain the wild type reporter, VEGFA 3'UTR-WT, and the mutant reporter, VEGFA 3'UTR-Mut.Cells ( $1 \times 10^{5}$ cells/well) were transfected with $50 \mathrm{ng}$ vector, $10 \mathrm{nM}$ miR-29c-3p, and $1 \mu \mathrm{l}$ Lipofectamine ${ }^{\circledR} 2000$ (Thermo Fisher Scientific, Inc.) in $100 \mu 1$ Opti-Minimum Essential medium (Gibco; Thermo Fisher Scientific, Inc.). At $72 \mathrm{~h}$ post-transfection, luciferase activity of Firefly and Renilla was monitored using a Dual-Luciferase Reporter Assay System (Promega Corporation), according to the manufacturer's protocol. The final results were normalized to the Renilla luciferase and analyzed statistically.

Statistical analysis. All the above experiments were verified with triplicate repetition. The final data were analyzed statistically using SPSS 19.0 software (IBM Corp., Armonk, NY, USA). All data are presented in the form of the mean \pm standard deviation. Spearman's correlation analysis was used to analyze the correlation between VEGFA and miR-29c-3p. Comparisons between two groups were performed using a Student's t-test, and differences among three groups were analyzed via one-way analysis of variance followed by the Student-Newman-Keuls test. $\mathrm{P}<0.05$ was considered to indicate a statistically significant difference.

\section{Results}

miR-29c and VEGFA expression in NSCLC tumor tissues are negatively correlated. In order to examine the expression patterns of miR-29c and VEGFA in NSCLC tumor tissues, 30 specimens from NSCLC cases with their corresponding para-carcinoma normal tissues were analyzed using the RT-qPCR method. As presented in Fig. 1, the analytical results indicated a significant downregulation of miR-29c and a significant upregulation of VEGFA in NSCLC tumor tissues, compared with the corresponding normal tissues. A significant negative correlation between the expression levels of miR-29c and VEGFA was observed in NSCLC tumor tissues $(\mathrm{P}=0.005)$.

miR-29c expression in lung cancer cell lines and VEGFA expression following transfection. In order to select the NSCLC cell line with the lowest expression level of miR-29c-3p for further experiments, the RT-qPCR method was employed to compare miR-29c expression levels in the human NSCLC cell lines A549, NCI-H1299 and H1650. As presented in Fig. 2A, the NCI-H1299 cell line had the lowest relative expression level of $\mathrm{miR}-29 \mathrm{c}$, and was chosen for subsequent experiments. The relative expression of VEGFA in three groups of NCI-H1299 cells was measured following transfection. The results demonstrated no significant difference between the VEGFA expression levels of the control and NC groups (Fig. 2B and C). However, the results demonstrated an upregulation of the expression of miR-29c-3p (Fig. 2B) and a downregulation of the expression of VEGFA (Fig. 2C) in the miR-29c mimic group, which further confirmed the negative correlation between the expression levels of miR-29c and VEGFA.

miR-29c expression affects lung cancer cell proliferation and apoptosis. The influence of miR-29c expression on NSCLC cell proliferation and apoptosis was further investigated using an MTT assay and Annexin V-FITC apoptosis assay. From the apoptosis assay results (Fig. 3A), the cellular apoptosis rates of the control group, $\mathrm{NC}$ group and the miR-29c mimic group were demonstrated to be $4.67,9.13$ and $53.8 \%$, respectively. The cellular apoptosis rate of miR-29c mimic group was significantly higher compared with the other two groups (Fig. 3B). This significant difference indicated that the overexpression of miR-29c may facilitate NSCLC cellular apoptosis. The growth curves from the MTT assay illustrated the influence of miR-29c on NSCLC cell proliferation (Fig. 3C). At 48 h, there was a clear suppressive effect on cell viability in the miR-29c mimic group compared with the other two groups, meaning 
A

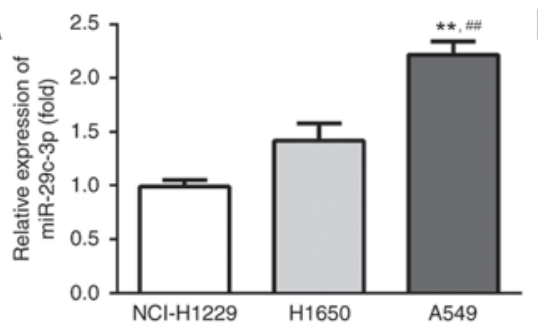

B

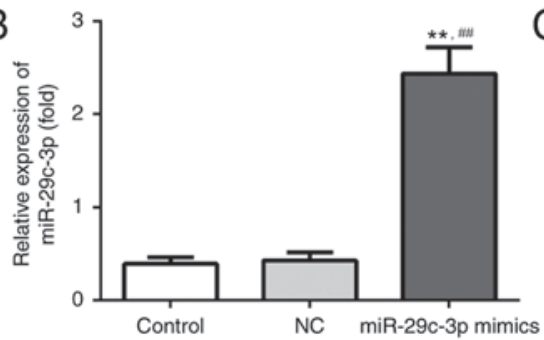

C

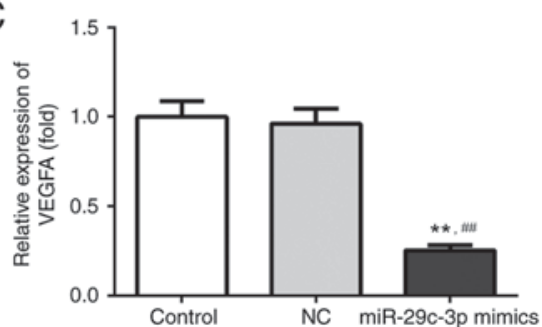

Figure 2. miR-29c-3p overexpression and VEGFA downregulation in lung cancer cell lines following miR-29c-3p mimics transfection. (A) miR-29c-3p expression levels in different lung cancer cell lines, and the expression levels of (B) miR-29c-3p and (C) VEGFA following transfection. ${ }^{* *} \mathrm{P}<0.01 \mathrm{miR}-29 \mathrm{c}-3 \mathrm{p}$ mimics vs. control group; ${ }^{\# \#} \mathrm{P}<0.01$ miR-29c-3p mimics vs. NC group. miR, microRNA; VEGFA, vascular endothelial growth factor A; NC, negative control.

A

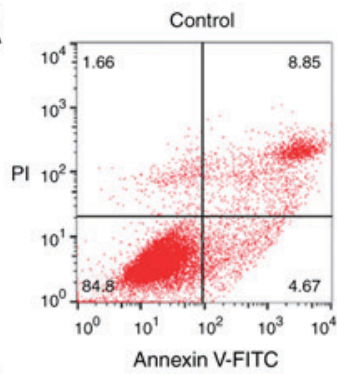

C

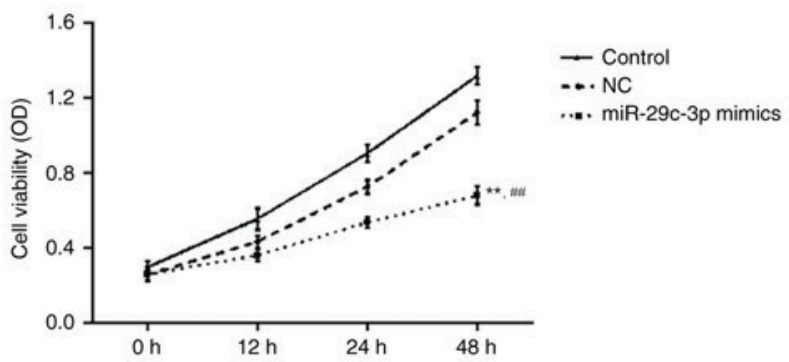

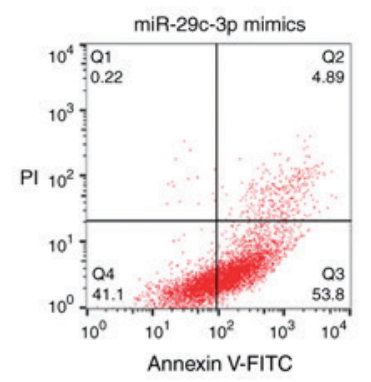

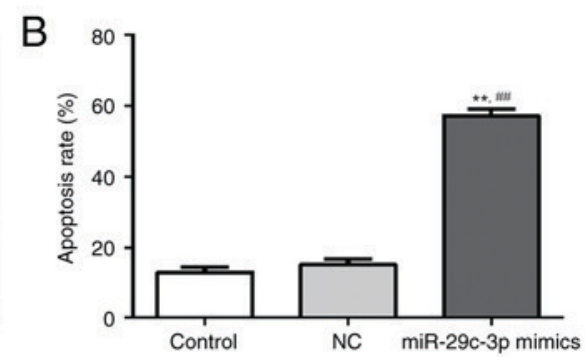

Figure 3. miR-29c-3p promotes cell apoptosis and inhibits cell proliferation of lung cancer cells. Cellular apoptosis analysis by (A) flow cytometry and (B) quantification, and (C) cell proliferation analysis in the three groups. ${ }^{* *} \mathrm{P}<0.01 \mathrm{miR}-29 \mathrm{c}-3 \mathrm{p}$ mimics vs. control group; ${ }^{\# \#} \mathrm{P}<0.01 \mathrm{miR}-29 \mathrm{c}-3 \mathrm{p}$ mimics vs. NC group. PI, propidium iodide; FITC, fluorescein isothiocyanate; NC, negative control; miR, microRNA; OD, optical density.

that the overexpression of miR-29c was able to inhibit NSCLC cell proliferation.

miR-29c regulates VEGFA expression and the PI3K/Akt signaling pathway in lung cancer cells. To further examine the association between the expression levels of miR-29c and VEGFA, and the possible regulatory mechanism of miR-29c through the PI3K/Akt signaling pathway, western blotting was used to measure the expression levels of VEGFA protein and other signaling pathway-associated proteins in the three groups. As presented in Fig. 4, no apparent variation was observed in the expression levels of VEGFA, p-PI3K and p-Akt proteins in the control and NC groups. However, in the miR-29c mimics group, the expression levels of VEGFA, p-PI3K and p-Akt proteins were significantly downregulated compared with the other two groups $(\mathrm{P}<0.01)$. This phenomenon indicated that miR-29c was able to regulate VEGFA expression and affect NSCLC cell activities via the PI3K/Akt signaling pathway.

VEGFA is a direct gene target of $m i R-29 c$. To verify the molecular basis for the regulatory role of miR-29c in NSCLC cell activities observed above, it was predicted that VEGFA may be a gene target for miR-29c using bioinformatics analysis
(Fig. 5A). Thereafter, dual-luciferase reporter analysis was used to confirm that VEGFA is a direct target for miR-29c. As presented in Fig. 5B, the relative luciferase activity in the WT VEGFA 3'UTR group was significantly decreased in miR-29c mimics-transfected cells compared with NC cells, while the relative luciferase activity in the mutant VEGFA 3'UTR group exhibited no notable decrease in cells transfected with miR-29c mimics. This result suggested that VEGFA may be a direct gene target of miR-29c.

\section{Discussion}

NSCLC has a median 5-year survival rate ranging between $51 \%$ (stage IA) and 26\% (stage IIIA), which is low compared with other common tumors, including breast cancer and prostate cancer (13). For early stage NSCLC, complete surgical resection along with cisplatin-based chemotherapy is the most frequently used therapeutic method, although the efficacy of chemotherapy remains uncertain (14). Notably, molecular targeted therapy for lung cancer has proven effective in dealing with specific resistant tumors and certain targeted agents that may inhibit EGFR have been approved for the treatment of NSCLC (15). Attention has 

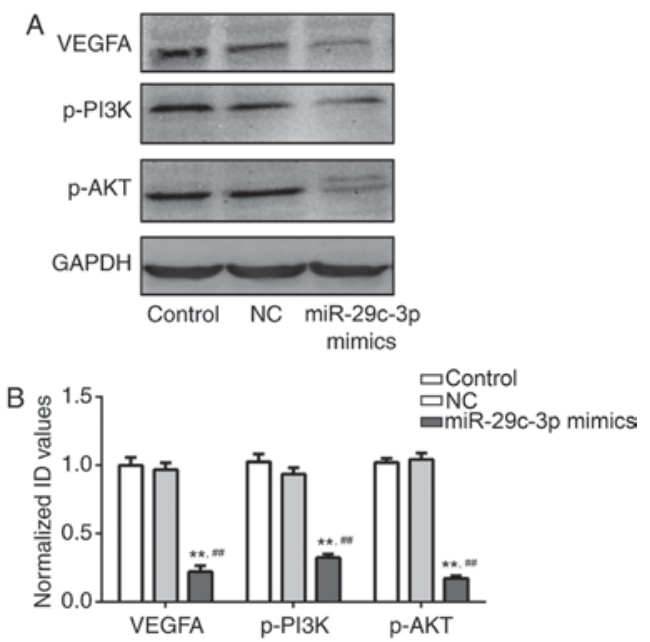

Figure 4. miR-29c-3p inhibits protein expression levels of VEGFA, $\mathrm{p}-\mathrm{PI} 3 \mathrm{~K} / \mathrm{p}-\mathrm{AKT}$ in lung cancer cells. (A) Western blot analysis of VEGFA and associated proteins in the three groups, and (B) quantification of the results. ${ }^{* *} \mathrm{P}<0.01$ miR-29c-3p mimics vs. control group; ${ }^{\# \prime} \mathrm{P}<0.01 \mathrm{miR}-29 \mathrm{c}-3 \mathrm{p}$ mimics vs. NC group. VEGFA, vascular endothelial growth factor A; ID, intensive density; p, phosphorylates; PI3K, phosphatidylinositol 3-kinase; AKT, RAC- $\alpha$ serine/threonine-protein kinase; $\mathrm{NC}$, negative control; miR, microRNA.

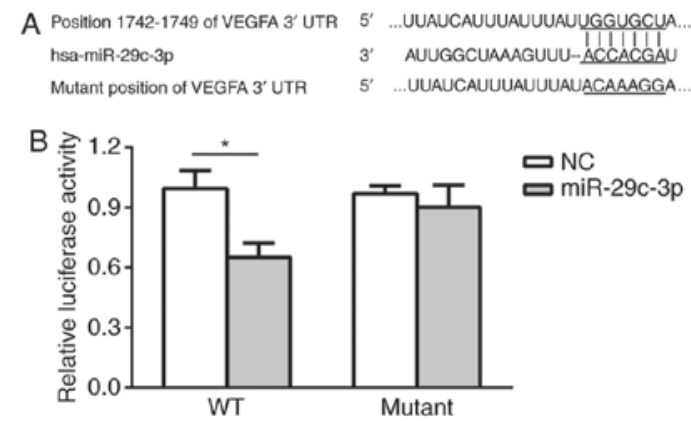

Figure 5. miR-29c-3p targets the $3^{\prime}$ UTR of VEGFA. (A) Bioinformatics results and (B) dual-luciferase reporter analysis results. ${ }^{*} \mathrm{P}<0.05 \mathrm{miR}-29 \mathrm{c}-3 \mathrm{p}$ mimics vs. NC group. VEGFA, vascular endothelial growth factor A; UTR, untranslated region; NC, negative control; WT, wild type; miR, microRNA.

been paid to the role of miRs as biomarker-driven targeted agents in various types of cancer (16). The miR-29 family, namely miR-29a, miR-29b and miR-29c, have been reported to be of importance in disease, including cardiac fibrosis, lung cancer, nasopharyngeal carcinoma and Alzheimer's disease (8,17-19). As mentioned previously, miR-29c has been proven to be a potential biomarker for the detection of early stage NSCLC and a promising targeted agent for the treatment of NSCLC $(20,21)$. However, the possible regulatory mechanism and corresponding signaling pathway of miR-29c in NSCLC tumor development remain to be elucidated. As an important factor in tumor growth, VEGFA is a possible target for miR-29c. A series of studies have reported the overexpression of VEGFA, which may contribute to poor prognosis in a range of types of cancer (22-24). Furthermore, it was reported that VEGFA was able to modulate the metastasis and invasion of lung cancer via the PI3K/Akt pathway (25). Based on this information, the present study investigated the correlation between miR-29c and VEGFA expression, and PI3K/Akt pathway-associated protein expression in NSCLC tumors, to elucidate the potential regulatory mechanism.

In the present study, the evident low expression of miR-29c was found in NSCLC tumor tissues in comparison to adjacent normal tissues. Correspondingly, the VEGFA expression level in NSCLC tumor tissues was upregulated compared with normal tissues. Statistical analysis demonstrated an evident negative correlation in the expression levels of miR-29c and VEGFA in NSCLC tumor tissues. To verify this observed negative correlation between miR-29c and VEGFA expression, further study was performed via transfection of miR-29c mimics into NSCLC tumor cells. It was confirmed that the overexpression of miR-29c was able to suppress VEGFA expression in the miR-29c mimics group compared with the other two control groups. Subsequently, analysis of the influence of miR-29c on NSCLC cell activities demonstrated that overexpression of miR-29c was able to inhibit the proliferation and promote the apoptosis of NSCLC cells. Since high expression of VEGFA has been reported to be correlated with the proliferation and metastasis of various types of tumor (11), it was hypothesized that miR-29c may regulate NSCLC cell activities by targeting VEGFA. Through bioinformatics analysis, it was predicted that VEGFA may be a direct target for miR-29c. The dual-luciferase reporter analysis demonstrated that miR-29c was able to bind the VEGFA 3'UTR directly and thus suppress VEGFA expression. Furthermore, western blot analysis results demonstrated that the expression level of VEGFA protein was downregulated in the miR-29c mimics group compared with the control groups. The expression levels of PI3K/Akt pathway-associated proteins, including $\mathrm{p}-\mathrm{PI} 3 \mathrm{~K}$ and p-Akt, were downregulated in the miR-29c mimics group. These results revealed for the first time, to the best of our knowledge, that miR-29c was able to modulate NSCLC tumor activity, including proliferative and apoptotic processes, by targeting VEGFA.

In conclusion, the functional role and possible mechanism of miR-29c in NSCLC tumor progression were investigated in the present study. Overall, it was demonstrated that miR-29c was aberrantly underexpressed in NSCLC tumor tissues, and there was an evident negative correlation between the expression levels of miR-29c and VEGFA. In particular, the overexpression of miR-29c was able to downregulate the expression level of VEGFA and decrease the proliferation, as well as promoting the apoptosis, of NSCLC tumor cells. Further experiments confirmed that VEGFA was a direct target of miR-29c, and that miR-29c was able to affect NSCLC tumor cell activities by modulating the PI3K/Akt signaling pathway. Although more detailed analysis of the accurate regulatory mechanisms of miR-29c in NSCLC tumor progression are required, the present study identified an important miR and its functional role in NSCLC tumors, and provided a basis for future clinical therapeutic research into NSCLC tumors.

\section{Acknowledgements}

Not applicable.

\section{Funding}

No funding was received. 


\section{Availability of data and materials}

Not applicable.

\section{Authors' contribution}

SZ designed and performed the experiments and analyzed the data. $\mathrm{CW}$ performed the experiments and analyzed the data. FY designed the experiments and composed the manuscript.

\section{Ethics approval and consent to participate}

The present study was approved by the Ethics Committee of Linyi People's Hospital (Linyi, China). Written informed consent was obtained from all patients.

\section{Consent for publication}

Not applicable.

\section{Competing interests}

The authors declare that they have no competing interests.

\section{References}

1. Jemal A, Bray F, Center MM, Ferlay JJ, Ward E and Forman D: Global cancer statistics. CA Cancer J Clin 61: 69-90, 2011.

2. Hu Z, Chen X, Zhao Y, Tian T, Jin G, Shu Y, Chen Y, Xu L, Zen K, Zhang C and Shen H: Serum microRNA signatures identified in a genome-wide serum microRNA expression profiling predict survival of non-small-cell lung cancer. J Clin Oncol 28 1721-1726, 2010.

3. Peters S, Adjei AA, Gridelli C, Reck M, Kerr K and Felip E; ESMO Guidelines Working Group: Metastatic non-small-cell lung cancer: ESMO clinical practice guidelines for diagnosis, treatment and follow-up. Ann Oncol 23 (Suppl 7): vii56-vii64, 2012.

4. Giovannetti E, Toffalorio F, De Pas T and Peters GJ Pharmacogenetics of conventional chemotherapy in non-small-cell lung cancer: A changing landscape? Pharmacogenomics 13 1073-1086, 2012.

5. Lin S and Gregory RI: MicroRNA biogenesis pathways in cancer. Nat Rev Cancer 15: 321-333, 2015.

6. Osada $\mathrm{H}$ and Takahashi T: MicroRNAs in biological processes and carcinogenesis. Carcinogenesis 28: 2-12, 2007.

7. Fabbri M, Garzon R, Cimmino A, Liu Z, Zanesi N, Callegari E, Liu S, Alder H, Costinean S, Fernandez-Cymering C, et al: MicroRNA-29 family reverts aberrant methylation in lung cancer by targeting DNA methyltransferases $3 \mathrm{~A}$ and 3B. Proc Natl Acad Sci USA 104: 15805-15810, 2007.

8. Zhang HW, Wang EW, Li LX, Yi SH, Li LC, Xu FL, Wang DL, Wu YZ and Nian WQ: A regulatory loop involving miR-29c and Sp1 elevates the TGF- $\beta 1$ mediated epithelial-to-mesenchymal transition in lung cancer. Oncotarget 7: 85905-85916, 2016.

9. Gu A, Lu J, Wang W, Shi C, Han B and Yao M: Role of miR-497 in VEGF-A-mediated cancer cell growth and invasion in non-small cell lung cancer. Int J Biochem Cell Biol 70: 118-125, 2016.
10. Claesson-Welsh L and Welsh M: VEGFA and tumour angiogenesis. J Intern Med 273: 114-127, 2013.

11. Zhang Y, Liu X, Zhang J, Li L and Liu C: The expression and clinical significance of PI3K, pAkt and VEGF in colon cancer. Oncol Lett 4: 763-766, 2012.

12. Livak KJ and Schmittgen TD: Analysis of relative gene expression data using real-time quantitative PCR and the 2(-Delta Delta $\mathrm{C}(\mathrm{T}))$ method. Methods 25: 402-408, 2001

13. Chuang JC, Neal JW, Niu XM and Wakelee HA: Adjuvant therapy for EGFR mutant and ALK positive NSCLC: Current data and future prospects. Lung Cancer 90: 1-7, 2015.

14. NSCLC Meta-analyses Collaborative Group1, Arriagada R, Auperin A, Burdett S, Higgins JP, Johnson DH, Le Chevalier T, Le Pechoux C, Parmar MK, Pignon JP, et al: Adjuvant chemotherapy, with or without postoperative radiotherapy, in operable non-small-cell lung cancer: Two meta-analyses of individual patient data. Lancet 375: 1267-1277, 2010.

15. Thomas A, Liu SV, Subramaniam DS and Giaccone G: Refining the treatment of NSCLC according to histological and molecular subtypes. Nat Rev Clin Oncol 12: 511-526, 2015.

16. Tibaldi C, D'Incecco A and Lagana A: MicroRNAs and targeted therapies in non-small cell lung cancer: Minireview. Anticancer Agents Med Chem 15: 694-700, 2015.

17. Van Rooij E, Sutherland LB, Thatcher JE, DiMaio JM, Naseem RH, Marshall WS, Hill JA and Olson EN: Dysregulation of microRNAs after myocardial infarction reveals a role of miR-29 in cardiac fibrosis. Proc Natl Acad Sci USA 105: 13027-13032, 2008.

18. Qiu F, Sun R, Deng N, Guo T, Cao Y, Yu Y, Wang X, Zou B, Zhang S, Jing T, et al: miR-29a/b enhances cell migration and invasion in nasopharyngeal carcinoma progression by regulating SPARC and COL3A1 gene expression. PLoS One 10: e0120969, 2015.

19. Lei X, Lei L, Zhang Z, Zhang Z and Cheng Y: Downregulated miR-29c correlates with increased BACE1 expression in sporadic Alzheimer's disease. Int J Clin Exp Pathol 8: 1565-1574, 2015.

20. Zhu W, He J, Chen D, Zhang B, Xu L, Ma H, Liu X, Zhang Y and Le H: Expression of miR-29c, miR-93, and miR-429 as potential biomarkers for detection of early stage non-small lung cancer. PLoS One 9: e87780, 2014.

21. Wang H, Zhu Y, Zhao M, Wu C, Zhang P, Tang L, Zhang H, Chen X, Yang Y and Liu G: miRNA-29c suppresses lung cancer cell adhesion to extracellular matrix and metastasis by targeting integrin $\beta 1$ and matrix metalloproteinase2 (MMP2). PLoS One 8: e70192, 2013.

22. Goos JA, de Cuba EM, Coupé VM, Diosdado B, Delis-Van Diemen PM, Karga C, Beliën JA, Menke-Van der Houven van Oordt CW, Geldof AA, Meijer GA, et al: Glucose transporter 1 (SLC2A1) and vascular endothelial growth factor a (VEGFA) predict survival after resection of colorectal cancer liver metastasis. Ann Surg 263: 138-145, 2016.

23. Mao D, Zhang Y, Lu H and Zhang H: Molecular basis underlying inhibition of metastasis of gastric cancer by anti-VEGFa treatment. Tumor Biol 35: 8217-8223, 2014

24. Geng L, Chaudhuri A, Talmon G, Wisecarver JL and Wang J: TGF-beta suppresses VEGFA-mediated angiogenesis in colon cancer metastasis. PLoS One 8: e59918, 2013.

25. Chen CH, Lai JM, Chou TY, Chen CY, Su LJ, Lee YC, Cheng TS, Hong YR, Chou CK, Whang-Peng J, et al: VEGFA upregulates FLJ10540 and modulates migration and invasion of lung cancer via PI3K/AKT pathway. PLoS One 4: e5052, 2009. 